Internist 2011 · 52:643-644

DOI 10.1007/s00108-011-2848-x

Online publiziert: 18. Mai 2011

(c) Springer-Verlag 2011
E. Märker-Hermann ${ }^{1} \cdot$ W.L. Gross ${ }^{2}$

${ }^{1}$ Klinik Innere Medizin IV, HSK Dr. Horst Schmidt Kliniken GmbH, Wiesbaden

2 Poliklinik für Rheumatologie, Universitätsklinikum Schleswig-

Holstein, Campus Lübeck und Klinikum Bad Bramstedt, Lübeck

\title{
Therapiestrategien in der Rheumatologie
}

\author{
Neue Konzepte, neue Substanzen, \\ Berücksichtigung von Komorbiditäten
}

Die Behandlung rheumatischer Erkrankungen hat sich in den vergangenen Jahren in nahezu allen Bereichen der entzündlichen Systemerkrankungen mit neuen Konzepten und innovativen Substanzen weiterentwickelt. Der aktuelle Themenschwerpunkt in Der Internist widmet sich mit der hier vorliegenden Ausgabe diesen neuen Therapiekonzepten bei der rheumatoiden Arthritis (RA), den Spondyloarthritiden, den mit antineutrophilen zytoplasmatischen Antikörpern (ANCA) assoziierten Vaskulitiden und auch neuen Konzepten bei der (degenerativen) erosiven Polyarthrose. Für den Internisten ist zudem in der Betreuung der Rheumapatienten von großer Bedeutung, die Komorbiditäten und möglichen therapiebedingten Komplikationen zu kennen, zu diagnostizieren und zu behandeln.

Am Beispiel der häufigsten entzündlich-rheumatischen Krankheiten, nämlich der RA und der Spondyloarthritiden, lässt sich anschaulich darstellen, dass mit den klinischen Studien, die zur Einführung der neuen Medikamente - im Wesentlichen der Biologika - führten, auch multinationale Initiativen zur Epidemiologie, Frühdiagnose, neuen bildgebenden Verfahren, zu prognostischen Faktoren und zu gesundheitsökonomischen Aspekten gestartet wurden. Diese Entwicklung mündete schließlich bei der RA (2010) und den Spondyloarthritiden (axiale Spondyloarthritiden 2009, periphere Spondyloarthritiden 2011) in neu- en, international konsentierten Kriterien zur (Früh-)Klassifikation dieser Erkrankungen. Praxisorientierte publizierte Empfehlungen zum Management der RA und der Spondyloarthritiden bilden für den internistischen Rheumatologen die Grundlagen, nach einer frühen Diagnose rasche Therapieentscheidungen fällen zu können.

Wichtig ist die Erkenntnis, dass Therapieanpassungen bzw. -änderungen eng an der individuellen Krankheitsaktivität (engmaschige Kontrollen!) und an prognostischen Faktoren orientiert sein müssen. Ziele der Behandlung sind heute nicht mehr allein die Linderung von Schmerzen, Reduktion der Entzündung und Verhinderung von weiteren knöchernen Zerstörungen mit entsprechendem Funktionsverlust, sondern Erhalt der Lebensqualität und Arbeitsfähigkeit. Ziel ist eine Remission der entzündlichrheumatischen Erkrankung. Die individuelle Verlaufskontrolle der Patienten unter antirheumatischer Therapie erfolgt mittels Verwendung validierter Messinstrumente wie z. B. dem Disease Activity Score DAS28, dem Health Assessment Questionnaire HAQ und anderen.

\section{() Therapieziel ist heute eine Remission der entzündlich- rheumatischen Erkrankung}

Auch für das Management der Vaskulitiden großer, mittelgroßer und kleiner Ge- fäße existieren seit kurzem Empfehlungen der European League Against Rheumatism (EULAR). Erwartungsgemäß zeigt sich analog zu anderen chronischentzündlichen Systemerkrankungen, dass eine frühe Diagnose und ein frühzeitiger Therapiebeginn das Outcome von Vaskulitispatienten wesentlich bestimmen. Aufgrund der relativen Seltenheit und des bunten klinischen Bildes von Vaskulitiden gelingt eine frühe Diagnose in der Praxis leider nicht immer. Der Facharzt für Innere Medizin ist aber häufig erster Ansprechpartner der Betroffenen und sollte die frühen Symptome kennen. Bei den ANCA-assoziierten Vaskulitiden, die schwerpunktmäßig in dieser Ausgabe von Der Internist dargestellt werden, haben aktuelle klinische Studien zur lokalisierten Form des M. Wegener, zur Remissionsinduktion der schweren systemischen Verlaufsform und zur Erhaltungstherapie des M. Wegener unser therapeutisches Handeln in den vergangenen Monaten geprägt.

Den Herausgebern ist es ein Anliegen, neben den entzündlichen Systemerkrankungen auch das wachsende Problem der degenerativen Polyarthrosen, hier speziell der erosiven Fingerpolyarthrose zu thematisieren. Eine pathogenetisch orientierte „Basistherapie“ der Arthrosen existiert zwar noch nicht. Aktive Grundlagenforschung - auch durch internistische Rheumatologen - und neue Konzepte auf dem Gebiet der Osteoar- 
throsen lassen aber hier in Kürze neue Therapien erwarten.

Den Komorbiditäten rheumatischer Erkrankungen ist ein abschließender Beitrag dieses Schwerpunktheftes gewidmet. Komorbiditäten können durch die systemische Entzündungsaktivität der rheumatischen Krankheit selbst, durch Störungen der immunologischen Abwehr oder durch Folgen der antirheumatischen Therapie bedingt sein, aber auch unabhängig von der Grundkrankheit auftreten. Rheumapatienten weisen eine erhöhte kardiovaskuläre Morbidität und Mortalität sowie häufig eine erhöhte Disposition für Infektionen, Tumoren und Osteoporose auf. Die Komorbiditäten sollten daher bereits zum Zeitpunkt der Erstmanifestation rheumatischer Erkrankung durch gezieltes Screening diagnostiziert und auch behandelt werden. Es gilt, den an Rheuma erkrankten Patienten in jeder Krankheitsphase interdisziplinär internistisch zu begleiten.

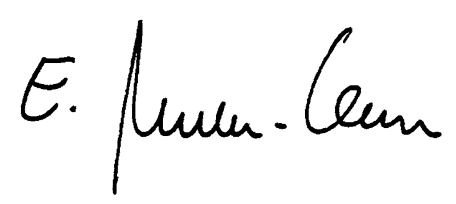

E. Märker-Hermann

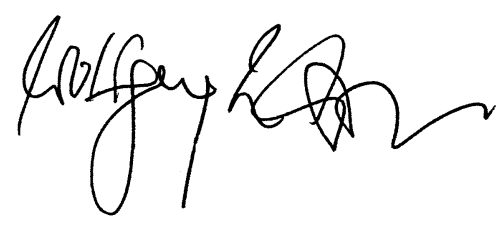

W.L. Gross

\section{Korrespondenzadresse}

\section{Prof. Dr. E. Märker-Hermann}



Interessenkonflikt. Die korrespondierende Autorin gibt an, dass kein Interessenkonflikt besteht.

\title{
Mehr Autonomie und engere Patientenbindung - ambulant und stationär wachsen zusammen
}

\author{
4. Deutscher Internistentag diskutiert Perspek- \\ tiven und Handlungsnotwendigkeiten für \\ Internisten in der sektorenübergreifenden \\ Versorgung
}

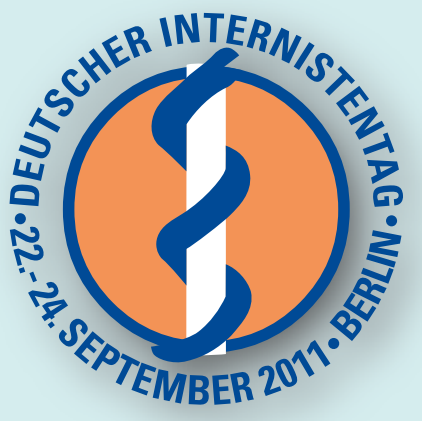

Mit dem Vertragsarztänderungsgesetz hat der Gesetzgeber ein wichtiges Zeichen gesetzt - die scharfe Abgrenzung zwischen ambulanten und stationären Versorgungsstrukturen ist durchlässig, die gleichzeitige Tätigkeit eines Facharztes in Praxis und Krankenhaus ermöglicht worden. Für den Arzt bedeutet dies eine stärkere berufliche Unabhängigkeit, der Patient darf mit einer qualitativen Verbesserung seiner Betreuung rechnen. Dennoch lässt sich für den heutigen Internisten sein berufliches Ziel einer medizinisch unabhängigen Tätigkeit im Rahmen der bestehenden Gesetzgebung weiterhin schwer realisieren.

Der Berufsverband Deutscher Internisten (BDI) erwartet darum vom Gesetzgeber weitere Regelungen, die die Auflösung der starren Trennung beider Versorgungsbereiche weiter vorantreiben. Der Verband fordert unter anderem, die Bedarfsplanung für neue Kooperationsstrukturen zu öffnen. Auf Seiten des Krankenhauses sei die ärztliche Zuständigkeit so zu regeln, dass der Konsiliararzt genauso wie der Belegarzt medizinisch unabhängig im Krankenhaus arbeiten könnten. Ebenfalls müssten die Leistungskataloge im Krankenhaus und in der Praxis soweit angeglichen werden, dass auch belegärztliche Leistungen abrechenbar sind, wenn sie nicht im EBM abgebildet sind.
Für die berufliche Zufriedenheit des Internisten hält es der BDI für unabdingbar, dass dieser Weg weiter gegangen wird. Darum lädt er auf dem 4. Deutschen Internistentag (22.-24. September 2011) in Berlin zu einer Podiumsdiskussion ein. Mit den Teilnehmenden diskutieren Prof. Dr. Wiedenmann, DGIM, Prof. Dr. Weiser, Verband leitender Krankenhäuser und Dr. Schmied, Vorstandsmitglied im BDI, unter Moderation von Dr. Spies, BDI. Die Veranstaltung verfolgt außerdem das Ziel, bei den betroffenen Vertragsund Krankenhausärzten das Bewusstsein dafür zu stärken, inwiefern bereits vorhandene gesetzliche Möglichkeiten besser genutzt werden können.

- Veranstaltung

"Zukunft des Internisten an der Schnittstelle ambulant/stationär" am Do., 22. September 2011 um 13.30 Uhr im Raum Virchow des Langenbeck-Virchow-Hauses, Berlin

Mehr unter: www.internistentag.de 